Nuclear Reactor Stability

By Dr. A. Hitchcock. (Nuclear Engineering Monographs, No. 11.) Pp. $x+61$. (London: Temple Press, Ltd., 1960.) 12s. 6d. net.

7 HE neutron multiplication in a reactor is affected by changes in temperature and other physical variables, which are in turn affected by changes in power. This circular situation can cause small changes in power-level to build up, so that the reactor becomes unstable. The possibility of unstable behaviour limits the available power output and must be either corrected by control action or, if possible, avoided by correct design. This monograph presents concisely, but with an admirably lucid and readable style, an introduction to the theory of reactor stability and the present methods used in establishing conditions for the stability of solid-and liquid-moderated power reactors, a theory which over the past fow years has led, ahead of experimental confirmation, to important advances in power reactor control. The treatment, avoiding the use of specialized techniques and the jargon of control system analysis, assumes only an elementary knowledge of linear differential equations and Fourier series.

The first two chapters show how the physical mechanism of several kinds of instability may be related to the partial differential equations of reactor dynamics. In the third and fourth chapters the modal expansion method is applied to yield conditions for instability, first due to temperature effects and then due to the relatively slow effect of fission product (xenon) poisoning. The last two chapters deal with voidage instability in boiling reactors and the principles of spatial control.

\section{Proceedings of the International Conference on} Nuclear Structure

Kingston, Canada, August 29-September 3, 1960. Edited by D. A. Bromley and E. W. Vogt. Pp. 990. (Toronto : University of Toronto Press ; Amsterdam : North-Holland Publishing Company, 1960.) $125 s$.

AREQUENT criticism of conference proceedings is that they tend to present a heterogeneous collection of papers which might more suitably and more speedily have been published in the appropriate technical journals. This criticism does not apply to the volume under review. Here we have a well. edited handbook of contemporary ideas on nuclear structure. It leads in logical sequence from a consideration of the properties of nuclear forces and infinite nuclear matter to a series of contributions on the detailed properties of levels in actual nuclei. The book is interfused throughout with contributions on the various reaction mechanisms by which our knowledge of nuclear structure is obtained. It is excellently produced with good, clear type and is well illustrated with diagrams reproduced from the contributors' slides. 'This is particularly praiseworthy because this book was available to the delegates within five weeks of the closing date of the confer. ence.

The editors have succeeded in effecting a pleasing compromise between the linguistic correctness of a submitted manuscript and the informality of expres. sion in the conference hall. This happy blend has been much aided by the inclusion of verbatim reports of the lively discussion which followed the main contributions. This book has been made what a good conference report should be. It provides those who were there with an accurate record of what was said, while at the same time it preserves something of the atmosphere of the conference hall for their less fortunate colleagues who were unable to be present.

D. L. Allan

\section{Progress in Dielectrics}

Vol. 3. Edited by Dr. J. B. Birks and Prof. J. Hart. Pp. vii +292 . (London : Heywood and Co., Ltd., 1961.) 63s. net.

THE third volume of this series is lively and well written. Three of the articles are concerned with electrical properties and molecular structure. R. H. Cole's review "Theories of dielectric relaxation and polarization" is helpful by its lucidity even when it covers old ground, and it contains some illuminating remarks, for example, on the dynamics of the Onsager equation. J. B. Hasted's article "The dielectric properties of water" (and ice) fills a long-felt want. This subject might deserve a book, particularly since it is relevant to biology. Hasted also gives a useful review of microwave techniques. R. J. Meakins systematically reviews new work on long-chain crystals, hydrogen-bonded compounds, occlusion compounds, and other types of solids the structure of which implies distinctive features of dielectric relaxation.

R. Stratton's review of theories of electrical breakdown is well presented, but this subject is perhaps being reviewed too frequently. The article by D. G. Kiely, "Dielectric waveguides and aerials", presents a mathematically difficult subject in a manner comprehensible to the experimentalist. The readable article by C. W. Hamilton on "Recent developments in cable insulation in the United States" is interesting in that it illustrates the gap which still exists between the questions asked by the engineer and the answers provided by the physicist and chemist.

V. DANIEL

Electrical Activity of Single Cells

Edited by Yasuji Katsuki. Pp. viii + 312. (Tokyo : Igaku Shoin, Ltd., 1960.) n.p.

7 HIS book consists of 18 separate articles by different workers in the field of electrophysiology. Their common feature, given by the editor as reason for grouping these articles together, is that they all represent work done in Japan with the intracellular capillary micro-electrode. The subjects of investigation include sensory receptors, peripheral and central nerve cells, the neuromuscular junction and cardiac muscle. Each article has the strictly limited range of interest of a paper in a scientific journal. Furthermore, apparently because the numerous authors were intent on presenting as much of their experimental results as space allowed, very little attention is given to describing the method or to justifying its application in different experimental situations. This is particularly unfortunate here, as it is the method which is the unifying feature of the book. A welcome exception to this is an article by Takeuchi and Takeuchi, who give a careful account of the application of the voltage-clamp technique to the localized response of the neuromuscular junction.

Much of the material in this book has already been published in readily accessible journals (in English), and it is clear that the remainder will soon follow. The book does not therefore fulfil any general need. P. FATT 